\title{
Acquired hypogammaglobulinemia in HIV-positive subjects after liver transplantation
}

\author{
K.S. Gregg, B. Barin, D. Pitrak, C. Ramaprasad, K. Pursell. Acquired \\ hypogammaglobulinemia in HIV-positive subjects after liver \\ transplantation. \\ Transpl Infect Dis 2013: 15: 581-587. All rights reserved
}

Abstract: Introduction. As more solid organ transplantations are performed in patients infected with human immunodeficiency virus (HIV), post-transplant complications in this population are becoming better defined.

Methods. Using serum samples from the Solid Organ Transplantation in HIV: Multi-Site Study, we studied the epidemiology of acquired hypogammaglobulinemia (HGG) after liver transplantation (LT) in 79 HIV-infected individuals with a median CD4 count at enrollment of 288 (interquartile range 200-423) cells $/ \mu \mathrm{L}$. Quantitative immunoglobulin $\mathrm{G}(\mathrm{IgG})$ levels before and after LT were measured, with moderate and severe HGG defined as IgG $350-500 \mathrm{mg} / \mathrm{dL}$ and $<350 \mathrm{mg} / \mathrm{dL}$, respectively. Incidence, risk factors, and associated outcomes of moderate or worse HGG were evaluated using KaplanMeier estimator and proportional hazards $(\mathrm{PH})$ models.

Results. The 1-year cumulative incidence of moderate or worse HGG was $12 \%$ (95\% confidence interval [CI]: 6-22\%); no new cases were observed between years 1 and 2 . In a multivariate $\mathrm{PH}$ model, higher pre-transplant model for end-stage liver disease score $(P=0.04)$ and treated acute rejection $(P=0.04)$ were both identified as significant predictors of moderate or worse HGG. There was a strong association of IgG levels $<500 \mathrm{mg} / \mathrm{dL}$ with non-opportunistic serious infection (hazard ratio [95\% CI]: $3.5[1.1-10.6] ; P=0.03$ ) and mortality (3.2 [1.1-9.4]; $P=0.04)$. These associations held after adjustment for important determinants of infection and survival among the entire cohort.

Conclusion. These results suggest that a proportion of HIV-positive LT recipients will develop clinically significant HGG after transplantation.

\author{
K.S. Gregg ${ }^{1}$, B. Barin ${ }^{2}$, D. Pitrak ${ }^{3}$, \\ C. Ramaprasad ${ }^{4}$, K. Pursell ${ }^{3}$
}

${ }^{1}$ Department of Internal Medicine, Division of Infectious Diseases, University of Michigan Medical School, Ann Arbor, Michigan, USA, ${ }^{2}$ EMMES Corporation, Rockville, Maryland, USA, ${ }^{3}$ Department of Internal Medicine, Section of Infectious Diseases and Global Health, University of Chicago Pritzker School of Medicine, Chicago, Illinois, USA, ${ }^{4}$ Department of Infectious Diseases, Kaiser Permanente Medical Group, San Jose, California, USA

Key words: HIV; liver transplantation;

hypogammaglobulinemia; infection; mortality

\section{Correspondence to:}

Kevin Gregg, Department of Internal Medicine,

Division of Infectious Diseases, University of

Michigan Medical School, 3119 Taubman Center,

1500 E. Medical Center Dr., SPC 5378, Ann Arbor,

MI 48109-5378, USA

Tel: (734) 2321486

Fax: (734) 9362737

E-mail: kvngregg@med.umich.edu

Received 1 August 2012, revised 29 January 2013,

2 April 2013, accepted for publication 9 April 2013

DOI: 10.1111/tid.12139

Transpl Infect Dis 2013: 15: 581-587
Since the introduction of highly active antiretroviral therapy (HAART) in 1996, the life expectancy of human immunodeficiency virus (HIV)-infected individuals in developed countries has increased greatly (1). In large part, because of this improved survival, HIV is no longer considered a contraindication to solid organ transplantation (SOT) (2). In this developing area of transplantation, the nature and outcomes of common post-transplant complications in HIV-infected individuals are still being defined.

One of these complications is acquired hypogammaglobulinemia (HGG), a well-described phenomenon after SOT (2). Patients require lifelong anti-rejection therapy after SOT, and many of the therapies used to achieve this have direct or indirect effects on humoral immune function. Reports in thoracic and abdominal organ transplantation have shown that a significant minority of recipients will develop clinically significant low immunoglobulin levels after SOT, and that this condition may predispose these subjects to adverse outcomes. In a cohort of 111 heart transplant recipients, $10 \%$ had immunoglobulin G ( $\mathrm{IgG}$ ) levels $<350 \mathrm{mg} / \mathrm{dL}$ after transplantation and were found to have a significantly increased risk of opportunistic infection 
compared with patients with higher IgG levels (3). Similarly, a single-center study of lung transplant recipients found $\mathrm{IgG}$ levels of $<400 \mathrm{mg} / \mathrm{dL}$ in $37 \%$ of recipients tested; affected subjects had an increased risk of bacterial, fungal, and tissue-invasive cytomegalovirus (CMV) disease compared with patients with IgG levels $>600 \mathrm{mg} / \mathrm{dL}$ (4). More recently, a cohort of kidney transplant recipients was found to have higher risk of bacteremia and acute pyelonephritis, if they developed HGG within 6 months of transplantation (5).

Recently, enrollment for the SOT in HIV: Multi-Site Study was completed. This study, which includes $>250$ patients, is investigating the safety and efficacy liver transplantation (LT) and kidney transplantation in HIVinfected persons with end-stage liver and/or kidney disease. Using stored serum and data from this study, we evaluated the incidence and associated adverse outcomes of HGG after SOT in this population and identified risk factors for development of this condition.

\section{Materials and methods}

We conducted a post-hoc analysis of prospectively collected serum samples and clinical data from the SOT in HIV: Multi-Site Study, which included 125 HIVinfected patients undergoing LT between October 2003 and February 2010 and was performed under the ClinicalTrials.gov number NCT00074386. For this study, institutional review board approval was granted by the University of Chicago Medical Center, Chicago, Illinois, USA (IRB: 10-477-A). The full inclusion and exclusion criteria have been previously published (6). Briefly, patients seeking LT needed to have a CD4+ T-cell count of $>100$ cells $/ \mu \mathrm{L}$ for 6 months and an undetectable viral load on a stable HAART regimen for 3 months prior to transplantation. Patients with a history of acquired immunodeficiency syndrome (AIDS)-defining opportunistic infections were excluded before April 2002. After that time, patients with treated opportunistic infections other than progressive multifocal leukoencephalopathy, chronic cryptosporidiosis, lymphoma, and visceral Kaposi's sarcoma were eligible for inclusion. Induction immunosuppression was not required but could be used at the discretion of the transplant team. All patients initially received corticosteroids and a calcineurin inhibitor (cyclosporine or tacrolimus) with or without mycophenolate mofetil. For opportunistic infection prevention, LT recipients received lifelong Pneumocystis jirovecii pneumonia prophylaxis with trimethoprim-sulfamethoxazole, dapsone, or atovaquone, and brief antifungal prophylaxis with fluconazole. Valganciclovir was given as secondary prophylaxis against CMV reactivation for 1 month after transplantation.

\section{Design}

Serum samples stored at $-80^{\circ} \mathrm{C}$ were used to conduct this study. Time point samples used in the analysis included the following: time 0 (pre-transplant), 3 months, 6 months, 1 year, and 2 years post transplant. Patients were excluded from analysis if no pretransplant or no post-transplant serum sample was available for analysis, or if they had preexisting HGG.

\section{Measurements}

Quantitative IgG levels were performed at the University of Chicago Medical Center core clinical laboratory. Samples were assayed using the Roche IgG-s Tinaquant $^{\circledR}$ platform (Roche Diagnostics, Indianapolis, Indiana, USA). HGG was defined as "moderate" if the IgG level was $350-500 \mathrm{mg} / \mathrm{dL}$ and "severe" if the $\operatorname{IgG}$ level was $<350 \mathrm{mg} / \mathrm{dL}$. These values have been suggested in previous publications and are similar to levels used by other studies $(2,4,7)$.

The primary study database was utilized to investigate the clinical and demographic risk factors for the development of HGG. Variables analyzed included the following: age, race, gender, body mass index (BMI) at enrollment $(<21)$, pre-transplant BMI $(<21)$, detectable HIV RNA at enrollment, CD4 count and nadir CD4 count at enrollment, hepatitis $\mathrm{C}$ virus (HCV) infection status, combined kidney-liver transplant, pre-transplant model for end-stage liver disease (MELD) score, donor age (by decade), basiliximab/daclizumab induction, initial tacrolimus therapy (vs. cyclosporine), mycophenolate mofetil administration, HAART regimen, ganciclovir/valganciclovir use, and receipt of hepatitis B immunoglobulin within 1 week of transplant. Baseline IgG level, as measured from the time 0 (pre-transplant) serum samples, was also included in this analysis. Post-transplant CD4 count and treated acute rejection (AR) were examined as time-dependent covariates.

Adverse events associated with HGG were also analyzed with the existing database and included allcause mortality, treated AR, and non-opportunistic serious infection in this analysis. Non-opportunistic serious infection was defined as an infection occurring during hospitalization or requiring hospitalization for treatment. Opportunistic serious infections were not included as there were too few (2 cases of esophageal 
candidiasis and 1 case of pneumocystosis) to perform any meaningful analysis.

\section{Statistical analysis}

Cumulative incidence of moderate or worse HGG was calculated with the Kaplan-Meier method, and 95\% confidence interval (CI) was estimated with the Greenwood's formula. Moderate or worse HGG was evaluated as a time-varying covariate in proportional hazards (PH) models for death, treated AR, and non-opportunistic serious infection. Risk factors for moderate or worse HGG were evaluated via PH models as well. All variables with $P<0.15$ from the univariate model were included in an initial multivariate model. Subsequently, variables with $P>0.1$ were excluded, the model was refit, and all interactions examined. A 2-sided $P$-value of $<0.05$ was considered to indicate statistical significance. Statistical analyses were performed with SAS software, version 9.2 (SAS Institute, Cary, North Carolina, USA).

\section{Results}

\section{Patient characteristics}

Of the 125 patients who underwent LT in the SOT in HIV: Multi-Site Study, 9 subjects had graft loss before 3 months and were excluded from analysis. Of the remaining 116 subjects, 81 had a pre-transplant and at least 1 post-transplant IgG level recorded. Two patients had pre-existing moderate $\mathrm{HGG}$ and were also excluded from analysis. Therefore, 79 LT recipients (including 4 liver-kidney recipients) were included in the analysis. Recipients were followed for a median of 3.0 (interquartile range [IQR] 2.0-4.5) years after transplant. Baseline demographic data of the included subjects were analyzed; 20\% were women, and $72 \%$ were white. Sixty-five percent developed end-stage liver disease because of HCV infection. Thirteen percent had a history of AIDS-related opportunistic infections or neoplasms. Subjects' HIV risk categories were men having sex with men $(42 \%)$, intravenous drug users (33\%), transfusion (9\%), heterosexual transmission (8\%), and unknown (9\%). Median baseline IgG was 1619 (IQR 1184-2267) mg/dL (Fig. 1). The median age at transplant was 48 (IQR 43-52) years, median pre-transplant MELD score was 18 (IQR 13-30), and median CD4 count at enrollment was 288 (IQR 200-423) cells/ $\mu$ L. Fifty-two percent received tacrolimus (vs. cyclosporine) as their initial calcineurin

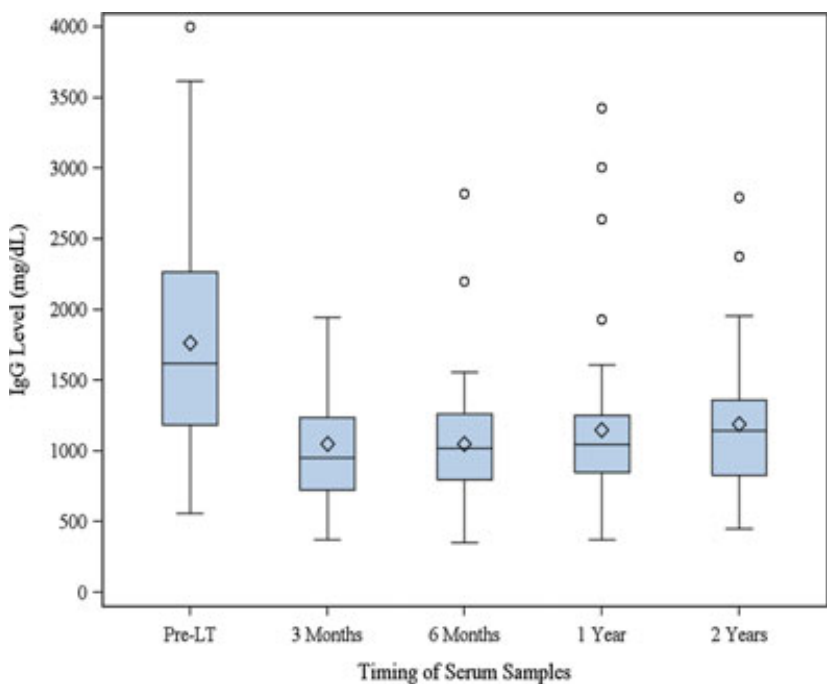

Fig. 1. Distribution of immunoglobulin G (IgG) levels pre-transplant and at select time points post liver transplant (LT).

inhibitor after transplant, and $29 \%$ received hepatitis B immunoglobulin within the first week after transplant because of hepatitis B co-infection. Fifty-four percent was on a protease inhibitor (PI)-based regimen shortly after transplant. Median donor risk index was 1.33 (IQR 1.16-1.63). Ninety-two percent of recipients were CMV seropositive and/or received an organ from a CMV-seropositive donor.

\section{Incidence of HGG}

Within 12 months of LT, 8 of 79 (10.1\%) recipients developed moderate HGG. No cases of severe HGG occurred in the same time period. No new cases of moderate or worse HGG were identified between years 1 and 2 post LT. Kaplan-Meier estimates for cumulative incidence of HGG at the data collection time points are shown in Table 1.

\begin{tabular}{|c|c|}
\hline Time point (post transplant) & Kaplan-Meier estimate (95\% CI) \\
\hline 3 months & $5 \%(2,13)$ \\
\hline 6 months & $10 \%(5,20)$ \\
\hline 1 year & $12 \%(6,22)$ \\
\hline 2 years & $12 \%(6,22)$ \\
\hline
\end{tabular}

Table 1 


\section{Outcomes associated with HGG}

The outcomes of interest in our study included mortality, non-opportunistic serious infection, and treated AR. Twenty-one subjects died post transplant: 7 before year 1,10 between years 1 and 3 , and 4 beyond year 3 . Reported causes of death were recurrent hepatitis C (in 5), malignancy (in 3), rejection (in 3), infection (in 2), multisystem organ failure (in 2), cardiac-related (in 2), and other/unknown (in 4). Of the 79 subjects, 42 developed a non-opportunistic serious infection. The first non-opportunistic serious infection post transplant was bacterial for 26 cases, fungal for 4 cases, viral for 4 cases, and of unknown source (no cultures or negative cultures) for 8 cases. The etiologic agents were Clostridium difficile (in 6), Enterococcus species (in 5), Staphylococcus aureus (in 4), Escherichia coli (in 3), Klebsiella species (in 3), Pseudomonas aeruginosa (in 2), Staphylococcus epidermidis (in 2), herpes zoster (in 2), and 1 each of Streptococcus species, Candida albicans, Torulopsis glabrata, Aspergillus fumigatus, Aspergillus species, CMV, and other virus. Overall, 29 subjects experienced treated AR: 27 first episodes were reported to be acute cellular rejection and 2 to be both acute cellular and acute vascular rejection.

In univariate analysis, moderate or worse HGG was associated both with non-opportunistic serious infection and death, but was not associated with treated AR (Table 2). Multivariate analysis included adjustment for important predictors of these outcomes including combined kidney-liver transplant, pre-transplant BMI

Association of moderate or worse hypogammaglobulinemia with adverse outcomes of infection, rejection, and death

\begin{tabular}{|c|c|c|}
\hline Adverse outcome & $\begin{array}{l}\text { Univariate analysis } \\
\text { HR ( } 95 \% \mathrm{Cl}, P \text {-value })\end{array}$ & $\begin{array}{l}\text { Multivariate analysis }{ }^{1} \\
\text { HR ( } 95 \% \mathrm{Cl}, P \text {-value) }\end{array}$ \\
\hline $\begin{array}{l}\text { Serious } \\
\text { non-opportunistic } \\
\text { infection }\end{array}$ & $\begin{array}{c}3.5(1.1-10.6 \\
P=0.03)\end{array}$ & $\begin{array}{c}5.2(1.7-16.4 \\
P=0.004)\end{array}$ \\
\hline $\begin{array}{l}\text { Treated acute } \\
\text { rejection }\end{array}$ & $\begin{array}{l}2.6(0.3-20.0 \\
P=0.37)\end{array}$ & $\mathrm{N} / \mathrm{A}$ \\
\hline Death & $\begin{array}{l}3.2(1.1-9.4 \\
P=0.04)\end{array}$ & $\begin{array}{c}3.2(1.1-9.7 \\
P=0.04)\end{array}$ \\
\hline
\end{tabular}

${ }^{1}$ Adjusted for significant predictors of serious non-opportunistic infection (post-transplant MELD score (time-varying), pre-transplant BMI, hepatitis C infection), and death (combined kidney-liver transplant, donor age, pre-transplant BMI, hepatitis C infection) among the entire cohort.

$\mathrm{HR}$, hazard ratio; $\mathrm{CI}$, confident interval; MELD, model for end-stage liver disease; N/A, not applicable; BMI, body mass index.

Table 2
$<21$, donor age (by decade), HCV infection, and posttransplant MELD score (time-varying covariate; serious infection model only). In this analysis, moderate or worse HGG continued to be significantly associated with non-opportunistic serious infection (hazard ratio [HR] 5.2 [95\% CI: 1.7-16.4]; $P=0.004$ ) and death (HR 3.2 [95\% CI: 1.1-9.7]; $P=0.04$ ).

\section{Risk factors for HGG}

Table 3 shows the univariate and multivariate analysis of selected predictors for the development of moderate or worse HGG. Age, baseline IgG level, pre-transplant MELD score, and treated AR were the only factors with $P<0.15$ from univariate analysis. In the final multivariate model, higher pre-transplant MELD score $(P=0.04)$ and treated AR $(P=0.04)$ were significantly associated with an increased risk of acquired HGG post LT, whereas older age was marginally associated with an increased risk of HGG $(P=0.06)$. Median pre-LT MELD score was 29 (IQR 19-38) in subjects with post-LT HGG and 18 (IQR 13-27) in those without.

\section{Discussion}

This is the first report, to our knowledge, of HGG after SOT in HIV-infected subjects. In this study, we found a $12 \%$ cumulative incidence of moderate or worse HGG at 1 and 2 years after LT among HIV-positive recipients. Patients who developed moderate or worse HGG were also found to have an increased risk of mortality and development of non-opportunistic serious infection.

The only previous study evaluating HGG and associated outcomes in LT recipients, to our knowledge, was performed in HIV-negative patients (7). In that study, 29 of 112 (26\%) patients developed IgG levels $<560 \mathrm{mg} / \mathrm{dL}$ within 32 weeks of LT; however, almost half of those patients had preexisting low IgG levels before transplant. The patients with HGG after transplant did not have any increased risk of infection or rejection compared with those with IgG levels $>560 \mathrm{mg} / \mathrm{dL}$, but they did have a significantly increased mortality at both 1 and 5 years. The only risk factors identified for developing HGG in this study were preexisting non-A/non-B hepatitis and "rare causes of hepatitis."

Our study found moderate HGG after LT in 8 of 79 HIV-infected patients for a cumulative incidence of $12 \%$ at 1 year post LT. This incidence is somewhat less than those found in previous studies involving non-HIVinfected heart, lung, and kidney transplant recipients, 
Analysis of possible risk factors for the development of moderate or worse hypogammaglobulinemia

\begin{tabular}{|c|c|c|}
\hline Univariate predictor & $\mathrm{HR}(95 \% \mathrm{CI})$ & $P$-value \\
\hline Age (by decade) & $1.8(0.9,3.9)$ & 0.12 \\
\hline Gender (female) & $0.6(0.1,5.3)$ & 0.69 \\
\hline Race (white) & $1.1(0.2,5.5)$ & 0.90 \\
\hline BMI at enrollment $<21$ & $1.1(0.1,9.1)$ & 0.92 \\
\hline Pre-transplant BMI <21 & $0.9(0.1,7.0)$ & 0.88 \\
\hline CD4 at enrollment (per 50 cells $/ \mu \mathrm{L}$ ) & $1.1(0.96,1.2)$ & 0.19 \\
\hline $\begin{array}{l}\text { Nadir CD4 at enrollment } \\
\text { (per } 50 \text { cells } / \mu \mathrm{L} \text { ) }\end{array}$ & $1.0(0.8,1.2)$ & 0.94 \\
\hline Detectable HIV RNA at enrollment & $2.1(0.4,10.4)$ & 0.36 \\
\hline HCV infection status (positive) & $0.9(0.2,3.8)$ & 0.89 \\
\hline Combined kidney-liver transplant & $3.5(0.4,28.5)$ & 0.24 \\
\hline Donor age (by decade) & $1.0(0.6,1.6)$ & 0.92 \\
\hline PI-based regimen as initial HAART & $2.7(0.5,13.2)$ & 0.23 \\
\hline $\begin{array}{l}\text { Tacrolimus as initial IS } \\
\text { (vs. cyclosporine) }\end{array}$ & $0.7(0.2,3.1)$ & 0.63 \\
\hline Initial mycophenolate mofetil use & $2.4(0.3-19)$ & 0.42 \\
\hline HBIg Induction & $1.5(0.4,6.2)$ & 0.60 \\
\hline Basiliximab/daclizumab induction & $1.2(0.2,10.2)$ & 0.84 \\
\hline Initial val/ganciclovir use & $1.2(0.1,9.7)$ & 0.87 \\
\hline Baseline IgG level (log-scale) & $0.1(0.002,2.0)$ & 0.12 \\
\hline Pre-transplant MELD score & $1.06(1.002,1.13)$ & 0.04 \\
\hline CD4 count $^{1}$ (per 50 cells $\left./ \mu \mathrm{L}\right)$ & $0.9(0.8,1.2)$ & 0.66 \\
\hline Treated acute rejection ${ }^{1}$ & $3.7(0.9,15)$ & 0.06 \\
\hline \multicolumn{3}{|l|}{ Multivariate predictors } \\
\hline Pre-transplant MELD score & $1.08(1.01,1.17)$ & 0.04 \\
\hline Treated acute rejection ${ }^{1}$ & $4.5(1.1,19)$ & 0.04 \\
\hline Age (by decade) & $2.0(0.97,4.1)$ & 0.06 \\
\hline \multicolumn{3}{|c|}{$\begin{array}{l}{ }^{1} \text { Time-varying covariate. } \\
\text { HR, hazard ratio; } \mathrm{CI} \text {, confidence interval; BMI, body mass index; HIV, } \\
\text { human immunodeficiency virus; HCV, hepatitis C virus; PI, protease } \\
\text { inhibitor; HAART, highly active antiretroviral therapy; IS, immuno- } \\
\text { suppression; HBIg, hepatitis B immunoglobulin; IgG, immunoglobulin } \\
\text { G; MELD, model for end-stage liver disease. }\end{array}$} \\
\hline
\end{tabular}

Table 3

where incidences have ranged from $26 \%$ to $45 \%(3,8,9)$. However, it is similar to the $13 \%$ incidence previously reported in HIV-negative LT recipients without preexisting HGG. Several explanations are possible for the lower incidence in this study, compared with those seen in previous studies. One explanation is the high median baseline IgG level of $1619 \mathrm{mg} / \mathrm{dL}$ in this cohort. It is reasonable to expect that an individual with a higher baseline IgG level may also have higher post-transplant IgG levels. Among previous SOT studies examining HGG, published baseline IgG levels have ranged from 1137 to $1281 \mathrm{mg} / \mathrm{dL}(3,8-10)$. In our cohort, liver disease and HIV both may have contributed to the high median pre-transplant IgG concentration. Hypergammaglobulinemia is well known to occur in many forms of chronic liver disease. Several mechanisms have been proposed as to why this occurs, including increased immune response to bacterial antigens from shunted portal blood flow and decreased catabolism of immunoglobulins by the liver $(11,12)$. Additionally, HIV infection frequently leads to polyclonal gammopathy with elevated IgG serum levels, which is thought possibly to be caused by a reduced number of memory B cells with concomitant hyperactivity of naïve B cells (13).

Another possible explanation for the low incidence of HGG in this study was the option for transplant teams to forego induction immunosuppression at the time of transplant. While it might be expected that patients receiving more intense immunosuppression would be at higher risk for developing $\mathrm{HGG}$, basiliximab or daclizumab induction was not found to be a significant risk factor for HGG in this study.

The most striking findings in our study were the significant associations of HGG with mortality and nonopportunistic serious infection. These associations remained present even when adjusted for important predictors of survival and infection among the entire cohort (14). This association with mortality is notable, as it has now been found in both studies examining HGG in LT recipients (7). The association of HGG and non-opportunistic serious infection raises a question as to the functionality of the IgG present in these patients. The IgG resulting from HIV gammopathy is composed of polyspecific self-reactive autoantibodies and nonspecific IgG (13); the increased rate of infection seen in these patients may indicate that they are deficient in antigen-specific antibody that contributes to infection prevention and clearance.

Pre-transplant MELD score and treated AR were the only clinical risk factors found to be associated with the development of HGG. Intensified immune suppression from anti-rejection treatment may likely be responsible for the low IgG state in these patients, and in fact, this association has been found previously in heart transplant recipients (3). It is not clear why a higher MELD score would predispose to developing HGG. Given that patients with advanced liver disease often have hypergammaglobulinemia, it might be expected that these patients would be at lower risk for HGG after transplantation. However, individuals with high MELD scores may have other clinical risk factors not captured 
in this study that increase the risk for HGG, such as protein-losing conditions that might predispose to HGG (15).

Similarly, owing to the small number of events and therefore limited power, other important risk factors might not have been detected with the current data. In particular, patients who develop HGG may have a genetic predisposition for this condition. Previous research has shown that genetic polymorphisms contribute to the pharmacokinetics and pharmacodynamics of common post-transplant immunosuppressant therapies such as mycophenolate mofetil and tacrolimus, suggesting that the degree of immune suppression may differ between patients receiving maintenance immunotherapy $(16,17)$. Further research involving gene- or micro-arrays would be necessary to further investigate this possibility.

Our study has several limitations. Overall, 35 of 114 patients were excluded from analysis because of lack of specimens, although no significant differences existed between excluded and included patients in baseline characteristics and post-transplant outcomes. Excluding these patients may have limited our ability to detect potential clinical and demographic predictors for developing HGG after transplant. In addition, the data from this study cannot elucidate causality. HGG may be a marker for overall degree of immunosuppression, thereby making adverse outcomes such as infection and death more likely, without actually contributing to either outcome.

In conclusion, our study provides the first evaluation, to our knowledge, of acquired HGG after LT in HIVpositive individuals. HIV-positive LT recipients without preexisting HGG have a similar incidence of development of HGG after transplant as do HIV-negative recipients, and appear to be most at risk within the first 12 months after transplant. These results are encouraging, as they do not suggest any additional risk for HGG in HIV-positive patients undergoing SOT, when compared with other recipient populations historically. However, recipients that do develop HGG appear to be at significantly greater risk of non-opportunistic serious infection and mortality than those who maintain adequate IgG levels. As high MELD score and treated AR appear to be risk factors for the development of HGG, these patients may be a population that should receive IgG level monitoring to detect HGG early, for possible treatment with supplemental immunoglobulins. Future prospective studies should investigate whether administration of intravenous or subcutaneous immunoglobulin in this population offers protection from the increased risk of infection and death in patients with an IgG measurement $<500 \mathrm{mg} / \mathrm{dL}$.

\section{Acknowledgement:}

We thank the Solid Organ Transplantation in HIV: Multi-Site Study (AI052748) that was funded by the National Institute of Allergy and Infectious Diseases, for supporting this research.

Disclosure: The authors of the manuscript have no associations that might pose a conflict of interest.

\section{References}

1. Antiretroviral Therapy Collaboration. Life expectancy of individuals on combination antiretroviral therapy in high-income countries: a collaborative analysis of 14 cohort studies. Lancet 2008; 372 (9635): 293-299.

2. Mawhorter S, Yamani MH. Hypogammaglobulinemia and infection risk in solid organ transplant recipients. Curr Opin Organ Transplant 2008; 13 (6): 581-585.

3. Yamani MH, Avery RK, Mawhorter SD, et al.

Hypogammaglobulinemia following cardiac transplantation: a link between rejection and infection. J Heart Lung Transplant 2001; 20 (4): 425-430.

4. Goldfarb NS, Avery RK, Goormastic M, et al. Hypogammaglobulinemia in lung transplant recipients. Transplantation 2001; 71 (2): 242-246.

5. Fernández-Ruiz M, López-Medrano F, Varela-Peña P, et al. Monitoring of immunoglobulin levels identifies kidney transplant recipients at high risk of infection. Am J Transplant 2012; 12 (10): 2763-2773.

6. Roland ME, Barin B, Carlson L, et al. HIV-infected liver and kidney transplant recipients: 1- and 3-year outcomes. Am J Transplant 2008; 8 (2): 355-365; erratum 8 (5): 1081.

7. Doron S, Ruthazer R, Werner BG, Rabson A, Snydman DR. Hypogammaglobulinemia in liver transplant recipients: incidence, timing, risk factors, and outcomes. Transplantation 2006; 81 (5): 697-703.

8. Yip NH, Lederer DJ, Kawut SM, et al. Immunoglobulin G levels before and after lung transplantation. Am J Respir Crit Care Med 2006; 173 (8): 917-921.

9. Keven K, Sahin M, Kutlay S, et al. Immunoglobulin deficiency in kidney allograft recipients: comparative effects of mycophenolate mofetil and azathioprine. Transpl Infect Dis 2003; 5 (4): 181-186.

10. Broeders EN, Wissing KM, Hazzan M, et al. Evolution of immunoglobulin and mannose binding protein levels after renal transplantation: association with infectious complications. Transpl Int 2008; 21 (1): 57-64.

11. Triger DR, Wright R. Hyperglobulinaemia in liver disease. Lancet 1973; 1 (7817): 1494-1496.

12. Tanaka S, Okamoto Y, Yamazaki M, Mitani N, Nakqjima Y, Fukui H. Significance of hyperglobulinemia in severe chronic liver diseases-with special reference to the correlation between serum globulin/IgG level and ICG clearance. Hepatogastroenterology 2007; 54 (80): 2301-2305.

13. De Milito A, Nilsson A, Titanji K, et al. Mechanisms of hypergammaglobulinemia and impaired antigen-specific humoral immunity in HIV-1 infection. Blood 2004; 103 (6): 2180-2186. 
14. Roland M. HIV-Related Predictors and Outcomes in 275 Liver and/or Kidney Transplant Recipients. ATC 2011, Philadelphia, PA, oral presentation, 2011.

15. Dahlqvist GE, Jamar F, Zech F, Geubel AP. In-111 transferrin scintigraphy in cirrhosis with hypoalbuminemia: evidence for protein-losing enteropathy in a small group of selected cases. Scand J Gastroenterol 2012; 47 (10): 1247-1252.
16. Lopez-Montenegro Soria MA, Kanter Berga J, Beltran Catalan S, Milara Paya J, Pallardo Mateu LM, Jimenez Torres NV. Genetic polymorphisms and individualized tacrolimus dosing. Transplant Proc 2010; 42 (8): 3031-3033.

17. Barraclough KA, Lee KJ, Staatz CE Pharmacogenetic influences on mycophenolate therapy. Pharmacogenomics 2010; 11 (3): 369-390. 